\title{
Hypoxia-induced circular RNA hsa_circ_0008450 accelerates hepatocellular cancer progression via the miR-431/AKAP1 axis
}

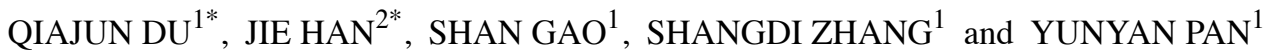 \\ ${ }^{1}$ Department of Clinical Laboratory Center, Lanzhou University Second Hospital; \\ ${ }^{2}$ Department of Endocrinology, The First Hospital of Lanzhou University, Lanzhou, Gansu 730030, P.R. China
}

Received January 2, 2020; Accepted August 4, 2020

DOI: 10.3892/ol.2020.12251

\begin{abstract}
Hypoxia facilitates the progression of numerous cancers. Circular RNAs (circRNA) have been revealed to be involved in the process of tumors mediated by hypoxia. However, the role and molecular mechanism of circular RNA hsa_circ_0008450 (circ_0008450) in hepatocellular cancer (HCC) under hypoxic conditions has been rarely reported. Expression levels of circ_0008450, microRNA(miR)-431 and A-kinase anchor protein 1 (AKAP1) were examined using reverse transcription-quantitative PCR. Cell viability, apoptosis and glycolysis were assessed via Cell Counting Kit-8, flow cytometry and glycolysis assays, respectively. The association between circ_0008450 or AKAP1 and miR-431 was verified via dual-luciferase reporter assays. Protein levels of AKAP1 were detected by western blotting. Effect of hsa_circ_0008450 on tumor growth in vivo was confirmed by xenograft assays. Circ_0008450 was upregulated in HCC tissues and hypoxia-disposed HCC cells. Depletion of circ_0008450 suppressed tumor growth in vivo and reversed the repression of apoptosis and the acceleration of viability and glycolysis of HCC cells induced by hypoxia treatment in vitro. Notably, circ_0008450 regulated AKAP1 expression by sponging miR-431. Furthermore, miR-431 inhibition reversed the circ_0008450 silencing-mediated effects on viability, apoptosis and glycolysis in hypoxia-treated HCC cells. Additionally, AKAP1 enhancement abolished the effects of miR-431 upregulation on the viability, apoptosis and glycolysis in hypoxia-treated HCC cells. In conclusion,
\end{abstract}

Correspondence to: Mr. Qiajun Du, Department of Clinical Laboratory Center, Lanzhou University Second Hospital, 82 Cuiyingmen, Lanzhou, Gansu 730030, P.R. China

E-mail: sw9mkj@163.com

*Contributed equally

Abbreviations: HCC, hepatocellular cancer; AKAP1, A-kinase anchor protein 1

Key words: hepatocellular cancer, circ_0008450, microRNA-431, A-kinase anchor protein 1, hypoxia circ_0008450 repression mitigated the progression of HCC under hypoxia by downregulating AKAP1 via miR-431, providing a potential target for HCC treatment.

\section{Introduction}

Hepatocellular cancer (HCC) is one of the most common invasive malignancies, ranking 4th among cancer-related causes of death in the world in 2018 (1). Despite advances in diagnostic and treatment strategies, the prognosis for $\mathrm{HCC}$ remains poor (2). The high proliferation and invasion activities of HCC cells are the main reasons for the poor prognosis of patients with HCC (3). The hypoxic characteristic of solid tumor tissue is caused by the rapid growth of the tumor and relatively low density of microvessels (4). It has been reported that hypoxia is involved in the angiogenesis, metastasis, proliferation, and chemotherapy resistance in patients with cancer (5-7). For instance, hypoxia-induced miR-182 accelerates the angiogenesis of HCC cells by downregulating Ras GTPase-activating protein 1 (5). Moreover, hypoxia-induced circular RNA (circRNA) circELP3 facilitates cisplatin resistance and tumor growth in bladder cancer (7). Though hypoxia exerts vital roles in the progression of $\mathrm{HCC}$, its potential molecular mechanisms remain unclear.

CircRNAs are a novel type of RNA with a closed circular structure (8). They regulate gene expression by modulating protein, miRNA and transcription functions (9). Moreover, circRNAs were revealed to be associated with the carcinogenesis of a range of malignant tumors (10). For example, circRNA circNT5E promotes the tumorigenesis of glioblastoma through sponging miR-422a (11). circRNA circPRKCI accelerates lung adenocarcinoma growth by elevating E2F7 expression by sponging miR-589 and miR-545 (12). Furthermore, circRNAs were involved in hypoxia-mediated cancer progression (13-15). Circular RNA hsa_circ_0008450 (circ_0008450), located at chromosome 16, is formed by the reverse splicing of the CMTM3 [CKLF-like MARVEL transmembrane domain containing (CMTM) 3] gene (from exon 3 to exon 5). Furthermore, it has been reported that circ_0008450 was associated with the development of nasopharyngeal cancer (16). Additionally, circ_0008450 may be acting as a potential prognostic marker for HCC (17). However, it remains unclear whether circ_0008450 is involved in the malignant behavior of $\mathrm{HCC}$ under hypoxic conditions. 
MicroRNAs (miRNAs or miRs) are a type of key regulator of gene expression and mainly regulate gene expression at the post-transcriptional level (18). They are involved in cell differentiation and metabolism, organismal development, oncogenesis and viral infection (19). miR-431 has been reported to act as a suppressor in multiple cancers, including pancreatic cancer (20), colorectal cancer (21) and lung cancer (22). Additionally, miR-431 participated in the advancement of HCC (23). However, whether miR-431 mediated the development of HCC under hypoxic conditions has not been reported.

A-kinase anchor protein 1 (AKAP1) is a member of the A-kinase Ankyrin family and serves important roles in intracellular cAMP signal transmission to the outer mitochondrial member (24). Moreover, AKAP1 regulated oxidative metabolism, organelle biogenesis and cell survival (25). Additionally, AKAP1 regulated endothelial cells behavior and vascular function (26). High AKAP1 expression predicted poor prognosis of HCC (27). However, the molecular mechanism of AKAP1 involved in the malignant behavior of HCC under hypoxic conductions needs to be further investigated.

The current study aimed to investigate the impacts of circ_0008450 on viability, apoptosis and glycolysis of HCC cells under hypoxic conductions.

\section{Materials and methods}

Study subjects. The current study was authorized by the Ethics Committee of Lanzhou University Second Hospital, Lanzhou, China. A total of 30 paired HCC tissues and corresponding adjacent normal tissues ( $>2.0 \mathrm{~cm}$ from the tumor margin) were obtained from patients with $\mathrm{HCC}$ who underwent resections at Lanzhou University Second Hospital. The clinicopathological features of HCC patients were presented in Table I. Moreover, serum samples were drawn from 30 patients with HCC and 30 healthy volunteers from the same hospital. All subjects were recruited between April 2017 and June 2019. The inclusion criteria for patients with HCC: i) Diagnosed with HCC and no other tumors and ii) without any treatment before surgery. The inclusion criteria for healthy volunteers were healthy individuals who were age-matched with the patients with HCC and had never been diagnosed with cancer. Written informed consent was obtained from patients with HCC and healthy volunteers.

Cell culture and treatment. HCC cell lines SNU-387 and Huh7, and human normal liver epithelial cells THLE-2, were purchased from BeNa Culture Collection. All cells were cultivated in RPMI-1640 medium (Sigma-Aldrich; Merck KGaA) supplemented with 10\% FBS (Sigma-Aldrich; Merck KGaA) and $1 \%$ penicillin/streptomycin stock solution (Sigma-Aldrich; Merck $\mathrm{KGaA}$ ) in a humidified atmosphere with $5 \% \mathrm{CO}_{2}$ at $37^{\circ} \mathrm{C}$. For the hypoxia treatment, SNU-387 and Huh7 cells were incubated in a humidified atmosphere with $94 \% \mathrm{~N}_{2}$, $5 \% \mathrm{CO}_{2}$ and $1 \% \mathrm{O}_{2}$ for $48 \mathrm{~h}$.

Cell transfection. Small interference RNA targeting circ_0008450(si-circ_0008450,5'-ACAAGACAGAAGGGTC TCTCA-3') and negative controls (si-NC, 5'-GCGCGATAGCG CGAATATA-3') were purchased from Guangzhou RiboBio Co., Ltd. miR-431 mimics (3'-ACGUACUGCCGGACGUUC UGU-5'), inhibitors (3'-ACAGAACGTCCGGCAGTACGT-5') and their negative controls (miRNA NC and inhibitor NC) were obtained from Shanghai GenePharma, Co., Ltd. The full-length sequences of circ_0008450 and AKAP1 were cloned into the pcDNA3.1 vector (vector; Invitrogen; Thermo Fisher Scientific, Inc.) or pCD5-ciR vector (pc-NC; Greenseed Biotech, Co.) to obtain the overexpression vectors for circ_0008450 and AKAP1, respectively. Oligonucleotides or vectors were transiently transfected into SNU-387 and Huh7 cells using the Lipofectamine 3000 reagent (Invitrogen; Thermo Fisher Scientific, Inc.). The transfection concentration of oligonucleotides was as follows: si-NC (40 nM), si-circ_0008450 (40 nM), miR-431 Mimics (50 nM), miRNA $\mathrm{NC}(50 \mathrm{nM}), \mathrm{miR}-431$ inhibitors $(50 \mathrm{nM})$, inhibitor NC $(50 \mathrm{nM})$. After transfection, cells were treated under hypoxia conditions for $48 \mathrm{~h}$.

Reverse transcription-quantitative PCR (RT-qPCR). Total RNA from tissues, serum and cells was extracted using the TRIzol $^{\circledR}$ reagent (Invitrogen; Thermo Fisher Scientific, Inc.). cDNA for circ_0008450, CMTM3, AKAP1 and miR-431 was generated using a Moloney Murine Leukemia Virus (M-MLV) First Strand kit (Thermo Fisher Scientific, Inc.) or a miRNA Reverse Transcription kit (Takara Biotechnology Co., Ltd.) according to the manufacturer's protocols. RT-qPCR was performed using SYBR Premix Ex Taq Takara Biotechnology Co., Ltd., with the following thermocycling conditions: $95^{\circ} \mathrm{C}$ for $30 \mathrm{sec}$, followed by 40 cycles of $95^{\circ} \mathrm{C}$ for $5 \mathrm{sec}$ and $60^{\circ} \mathrm{C}$ for $20 \mathrm{sec}$. The expression of circ_0008450, CMTM3, AKAP1, HIF-1 $\alpha$ and miR-431 was quantified using the $2^{-\Delta \Delta \mathrm{Cq}}$ method (28). Primers used were as follows: circ_0008450 forward (divergent), 5'-TGATGCATCCCATC CACCCT-3' and reverse (divergent), 5'-AGCCCTGCCACTTG TCATTC-3'; CMTM3 forward (convergent) 5'-TTTTATCTGC TATGTGGCGTCC-3' and reverse (convergent), 5'-TGTCTT GTGGGCTGTGGTCTC-3'; AKAP1 forward, 5'-GCTTAC GGCTTGTACCTGAAG-3' and reverse, 5'-ATGGTGCTCTTG GAAATACGC-3'; miR-431 forward, 5'-CAGGCCGTCATGC AAA-3' and reverse, 5'-CGCTTCAGAATTTGCGTGTCAT-3'; U6 small nuclear RNA (snRNA) forward, 5'-GCTCGCTTCGG CAGCACA-3' and reverse, 5'-GAGGTATTCGCACCAGA GGA-3'; HIF-1 $\alpha$ forward, 5'-TATGAGCCAGAAGAACTTTTA GGC-3'andreverse,5'-CACCTCTTTTGGCAAGCATCCTG-3'; GAPDH forward (convergent), 5'-GACTCCACTCACGGC AAATTCA-3' and reverse (convergent), 5'-TCGCTCCTGGAA GATGGTGAT-3'; and GAPDH forward (divergent), 5'-GAAGGTGAAGGTCGAGTC-3' and reverse (divergent), 5'-GAAGATGGTGATGGGATTTC-3'. Oligo(dt) ${ }_{18}$ primers (cat. no. SO132) and random primers (cat. no. 48190011) were bought from (Invitrogen; Thermo Fisher Scientific, Inc.). GAPDH or U6 snRNA served as an internal control. To verify the splicing from head-to-tail of circRNA, we designed divergent primers and convergent primers to amplify circRNA and linear RNA, and GAPDH was used as negative control.

Actinomycin D treatment and RNase R digestion. Huh7 cells were treated with $50 \mathrm{ng} / \mathrm{ml}$ Actinomycin D (Sigma-Aldrich; Merck $\mathrm{KGaA})$ at different times $(0,4,8,12$ or $24 \mathrm{~h})$ to block new RNA synthesis. Additionally, total Huh7 RNA was degraded with $3 \mathrm{U} / \mu \mathrm{g}$ RNase $\mathrm{R}$ (Epicentre; Illumina, Inc.) at $37^{\circ} \mathrm{C}$ for $15 \mathrm{~min}$. 
Table I. Association between circ_0008450 expression and clinical clinicopathological parameters of patients with hepatocellular cancer.

\begin{tabular}{|c|c|c|c|c|}
\hline \multirow[b]{2}{*}{ Parameter } & \multirow[b]{2}{*}{$\begin{array}{l}\text { Patients, } \\
\qquad \mathrm{n}\end{array}$} & \multicolumn{2}{|c|}{$\begin{array}{c}\text { circ_0008450 } \\
\text { expression }\end{array}$} & \multirow[b]{2}{*}{ P-value } \\
\hline & & $\begin{array}{l}\text { Low } \\
n=14\end{array}$ & $\begin{array}{l}\text { High } \\
n=16\end{array}$ & \\
\hline Age, years & & & & 0.07 \\
\hline$\leq 60$ & 14 & 9 & 5 & \\
\hline$>60$ & 16 & 5 & 11 & \\
\hline Sex & & & & 0.232 \\
\hline Female & 18 & 10 & 8 & \\
\hline Male & 12 & 4 & 8 & \\
\hline Tumor size, cm & & & & $0.003^{\mathrm{a}}$ \\
\hline$\leq 5$ & 17 & 12 & 5 & \\
\hline$>5$ & 13 & 2 & 11 & \\
\hline TNM stage & & & & $0.003^{\mathrm{a}}$ \\
\hline I-II & 15 & 11 & 4 & \\
\hline III & 15 & 3 & 12 & \\
\hline $\mathrm{AFP}, \mu \mathrm{g} / 1$ & & & & 0.696 \\
\hline$\leq 400$ & 16 & 8 & 8 & \\
\hline$>400$ & 14 & 6 & 8 & \\
\hline Lymphatic metastasis & & & & $0.0001^{\mathrm{a}}$ \\
\hline Negative & 12 & 11 & 1 & \\
\hline Positive & 18 & 3 & 15 & \\
\hline Vascular invasion & & & & 0.961 \\
\hline Absent & 13 & 6 & 7 & \\
\hline Present & 17 & 8 & 9 & \\
\hline Distant metastasis & & & & $0.0003^{\mathrm{a}}$ \\
\hline M0 & 15 & 12 & 3 & \\
\hline M1 & 15 & 2 & 13 & \\
\hline
\end{tabular}

${ }^{\mathrm{a}} \mathrm{P}<0.05$ as analyzed by $\chi^{2}$ tests. TNM, tumor, node and metastasis staging system; $\alpha$-fetoprotein tumor marker. M0, HCC was not diffused. M1, HCC had spread to other parts of the body.

Cell viability assay. Viability of transfected SNU-387 and Huh7 cells was assessed by Cell Counting kit-8 (CCK-8) assays. The cells were cultured under hypoxia conditions for $48 \mathrm{~h}$ after transfection. Cells were then incubated with $10 \mu 1$ CCK-8 reagent (Dojindo Molecular Technologies, Inc.) for $2 \mathrm{~h}$. Color reactions were determined using a Microplate Absorbance Reader (Bio-Rad Laboratories, Inc.).

Flow cytometry assay. Apoptosis rate of transfected and hypoxia-treated SNU-387 and Huh7 cells was evaluated using an Annexin V-FITC/propidium iodide (PI) apoptosis detection kit (Sigma-Aldrich; Merck KGaA) according to the manufacturer's protocols. Briefly, the cells were collected and resuspended in binding buffer and labeled with $5 \mu \mathrm{l}$ Annexin V-FITC and $10 \mu \mathrm{lPI}$ in the dark at room temperature for $15 \mathrm{~min}$. The apoptotic rate (early + late apoptotic cells) was analyzed using a FACScan flow cytometer (Becton-Dickinson and Company) with Cell Quest software (version 4.0.2, Becton-Dickinson and Company).

Measurement of glucose consumption and lactate production. Cell supernatants of transfected and hypoxia-treated SNU-387 and Huh7 cells were collected and the glucose consumption and lactate production of the cells were assessed using a Glucose Assay kit or a Lactic Acid Assay kit (both from Sigma-Aldrich; Merck KGaA) in accordance with the manufacturer's procedures.

Dual-luciferase reporter assay. Binding sites of miR-431 in circ_0008450 or AKAP1 were predicted using CircRNA Interactome (https://circinteractome.irp.nia.nih.gov/) or Starbase (http://starbase.sysu.edu.cn/) databases. Following this, fragments of circ_0008450 (position, 68-73; CAAGAC) or the 3'-untranslated region (UTR) of AKAP1 (position, 431-438; GCAAGAC; harbored miR-431 binding sites) was amplified and inset into the pGL3 vector (Promega Corporation) for the construction of wild-type luciferase reporter vectors. Mutant luciferase reporter vectors for circ_0008450 (GUUCUG) or AKAP1 (CGUUCUG; within miR-431 binding sites) were established using the same method. Luciferase reporter vectors and miRNA NC, miR-431 mimics, inhibitor NC or miR-431 inhibitors were cotransfected into 293T cells using Lipofectamine 3000 reagent (Invitrogen; Thermo Fisher Scientific, Inc.). After $48 \mathrm{~h}$, the luciferase intensities of the luciferase reporter vectors in 293T cells were determined using a luciferase reporter assay kit (Promega Corporation) based on the manufacturer's procedures. Relative activity was normalized to Renilla luciferase activity.

Western blotting. HCC cells were lysed using lysis buffer (Beyotime Institute of Biotechnology) to extract total protein and western blotting was conducted as previously described (29). A BCA ${ }^{\mathrm{TM}}$ Protein Assay kit (Pierce; Thermo Fisher Scientific, Inc.) was utilized to quantify the concentration of total protein. Briefly, 10\% SDS-PAGE was used to separate the total protein $(30 \mu \mathrm{g} / \mathrm{lane})$. Membranes with transferred proteins were blocked with TBST supplemented with 5\% BSA (Sigma-Aldrich; Merck KGaA). Primary antibodies used were as follows: rabbit anti-AKAP1 (cat. no. ab156004; 1:1,000; Abcam), rabbit anti-cleaved caspase-3 (caspase-3) (cat. no. ab2302; 1:300; Abcam), and rabbit-anti-GAPDH (1:10,000; cat. no. ab181602). Secondary antibodies used were goat anti-rabbit antibodies (cat. no. ab97051; 1:10,000; Abcam). Protein bands were visualized using ImmunoStar LD (Fujifilm Wako Pure Chemical Corporation) and GAPDH was used as the loading control. Densitometry analysis was performed using ImageJ software (version 1.6; National Institutes of Health).

Xenograft assay. Animal experiments were approved by the Animal Ethics Committee of Lanzhou University Second Hospital. A total of 8 female BALB/c nude mice (weight, 17-22 g; age, 4-6 weeks) were obtained from the Shanghai Experimental Animal Center for the xenograft assays. Briefly, PHBLV-U6-ZSGreen-puro lentivirus vectors (Hanbio Biotechnology Co., Ltd.) with short hairpin (sh)-circ_0008450 were infected into Huh7 cells for 1 week under puromycin. 
Following this, Huh7 cells $\left(5 \times 10^{6}\right)$ transfected with sh-NC or lentivirus-mediated sh-circ_0008450 were resuspended in $50 \mu 1$ PBS (Beijing Solarbio Science \& Technology Co., Ltd.) and subcutaneously injected into the dorsal side of nude mice (4 mice/group). All mice were kept in a specific pathogen-free room $(50 \pm 5 \%$ humidity) with a $12 \mathrm{~h}$ light $/ 12 \mathrm{~h}$ dark cycle at $25^{\circ} \mathrm{C}$ and free access to water and food. Tumor volumes were measured using a digital caliper every 4 days from day 7 post-injection and calculated using the following equation: Volume $=\left(\right.$ length $\mathrm{x}$ width $\left.{ }^{2}\right) / 2$. Mice were anesthetized with $10 \mathrm{mg} / \mathrm{kg}$ xylazine and euthanized by cervical decapitation on day 27 post-injection. Tumor tissues were excised after death as assessed by pupil dilation and cardiac arrest. Tumor tissues were weighed and then digested with TRIzol ${ }^{\circledR}$ reagent (Invitrogen; Thermo Fisher Scientific, Inc.) for the evaluation of the expression levels of circ_0008450,miR-431 and AKAP1.

Statistical analysis. Experiments were performed in triplicate. GraphPad Prism software (version 5.0; GraphPad Software, Inc.) and SPSS software (version 22.0; SPSS, Inc.) were used for statistical analysis. Data are presented as mean \pm standard deviation. Paired or unpaired Student's t-tests were performed to analyze the differences between two groups and ANOVA with Tukey's pos-hoc test was used to determine differences between $\geq 3$ groups. The association between circ_0008450 expression and clinical clinicopathological parameters of patients with HCC were determined using the $\chi^{2}$ test. Receiver operating characteristic curve (ROC) analysis was performed to analyze differences between patients with HCC and healthy controls. $\mathrm{P}<0.05$ was considered to indicate a statistically significant difference.

\section{Results}

Circ_0008450 is upregulated in the tissues and serum of patients with HCC patients and HCC cells. To verify the expression pattern of circ_0008450 in HCC, the expression of circ_0008450 in 30 paired HCC tissues and adjacent normal tissues were examined using RT-qPCR. The results demonstrated that circ_0008450 expression was significantly increased in HCC tissues compared with adjacent normal tissues (Fig. 1A). Furthermore, the expression of HIF-1 $\alpha$ was increased in HCC tissues compared with adjacent normal tissues (Fig. S1). The expression of circ_0008450 was significantly increased in the serum of patients with HCC patients compared with healthy controls (Fig. 1B). Moreover, ROC analysis demonstrated that circ_0008450 exhibited extremely high diagnostic value to distinguish patients with HCC from healthy controls (AUC=0.97; Fig. 1C). High circ_0008450 expression was associated with tumor size $(\mathrm{P}=0.003)$, TNM stage $(\mathrm{P}=0.003)$, lymphatic metastasis $(\mathrm{P}=0.0001)$ and distant metastasis $(\mathrm{P}=0.0003)$; however, circ_0008450 expression was not associated to age, sex, $\alpha$-fetoprotein (AFP) or vascular invasion (Table I). Furthermore, the expression of circ_0008450 was elevated in HCC cells (SNU-387 and Huh7) compared with THLE-2 cells (Fig. 1D). Circ_0008450 and linear GAPDH were amplified based on cDNA and genomic DNA (gDNA) using divergent and convergent primers. The results demonstrated that circ_0008450 was amplified by primers in cDNA; however, circ_000845 was not amplified by gDNA (Fig. S2). Subsequently, the circularization of circ_0008450 was further verified using Huh7 cells. Circ_0008450 was more stable and resistant to actinomycin D treatment compared with linear CMTM3 (Fig. 1E). There was no significant difference in circ_0008450 expression following RNase R treatment; however, the linear CMTM3 gene was digested (Fig. 1F). Furthermore, circ_0008450 expression was significantly decreased using oligo $(\mathrm{dt})_{18}$ primers compared with random primers, while the expression of CMTM3 did not exhibit a significant change, indicating that circ_0008450 did not have a ploy-A tail structure (Fig. 1G). These data indicated that circ_0008450 had high diagnostic value and its elevation may be associated with the progression of HCC.

Circ_0008450 depletion represses viability and glycolysis, and accelerates apoptosis of HCC cells following hypoxia treatment. Due to the upregulation of circ_0008450 in HCC, the role of circ_0008450 in HCC under hypoxia was investigated. RT-qPCR results revealed that circ_0008450 was significantly upregulated in SNU-387 and Huh7 cells treated with hypoxia compared with SNU-387 or Huh7 cells under normoxic conditions (Fig. 2A). Additionally, no significant change in THLE-2 cells. Regardless of whether the circ_0008450 gene was suppressed, the apoptosis and glycolysis of THLE-2 cells were not significantly different under normoxic or hypoxic conditions (Fig. S3). Hypoxia-induced enhancement of circ_0008450 in SNU-387 and Huh7 cells was abolished by the introduction of si-circ_0008450 (Fig. 2B). Additionally, the viability of SNU-387 and Huh7 cells was increased under hypoxia and this effect was reversed by circ_0008450 inhibition (Fig. 2C). The results of flow cytometry assays demonstrated that the promotive effect of hypoxia on the apoptosis of SNU-387 and Huh7 cells was abolished by circ_0008450 suppression (Fig. 2D and E). Furthermore, circ_0008450 knockdown partly reversed the downregulation of caspase-3 in SNU-387 and Huh7 cells following hypoxia treatment (Fig. S4). Additionally, the results of glycolysis assays demonstrated that glucose consumption (Fig. 2F) and lactate production (Fig. 2G) were increased in SNU-387 and Huh7 cells following hypoxia treatment. These effects were reversed by circ_0008450 knockdown.Collectively, these findings revealed that circ_0008450 silencing reverse cell viability, glycolysis and lactate production, and accelerated cell apoptosis in hypoxia-treated HCC cells.

Circ_0008450 targets miR-431 in hypoxia-treated HCC cells. To examine the regulatory mechanism of circ_0008450 in HCC, the underlying target for circ_0008450 was predicted. The CircRNA interactome database revealed that circ_0008450 had binding sites for miR-431 (Fig. 3A). The data revealed that miR-431 mimics significantly repressed the luciferase intensity of luciferase reporters containing WT-circ_0008450 in 293T cells compared with miRNA NC, while the luciferase intensity of luciferase reporters containing MUT-circ_0008450 was not significantly altered (Fig. 3B). Additionally, the luciferase activity of luciferase reporters containing WT-circ_0008450 in 293T was significantly increased by miR-431 inhibitors (Fig. 3C) and circ_0008450 expression was significantly elevated in SNU-387 and Huh7 cells following circ_0008450 transfection compared to control 
A

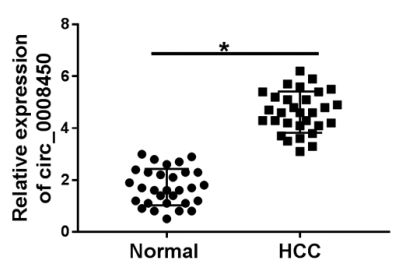

E

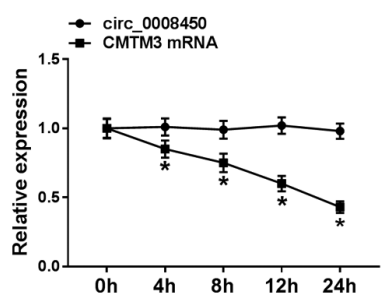

B

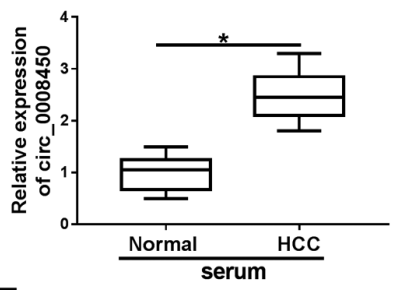

$\mathbf{F}$

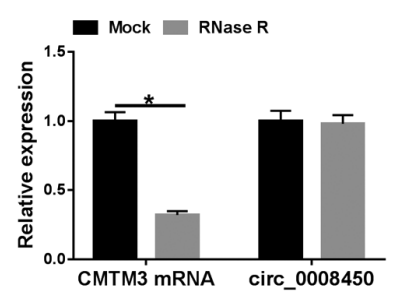

C

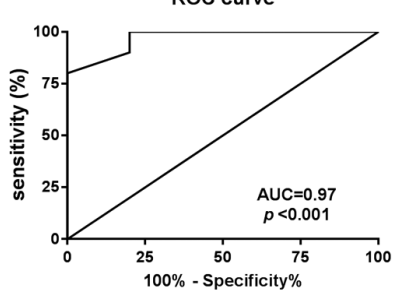

G

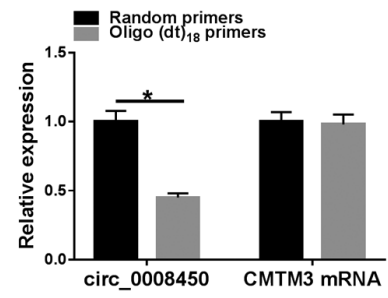

D

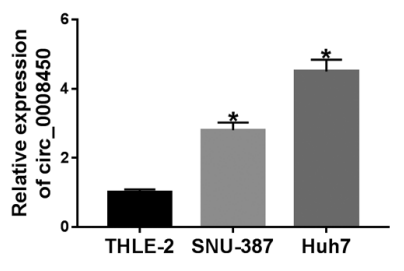

Figure 1.Expression pattern of circ_0008450 in the tissues and serum of patients with HCC and HCC cells. RT-qPCR was performed to detect the (A) expression of circ_0008450 in 30 paired HCC tissues and adjacent normal HCC tissues and to analyze the (B) expression of circ_0008450 in serum of patients with HCC and healthy controls. (C) ROC analysis was used to assess the diagnostic value of circulating circ_0008450 for differences between patients with patients and healthy controls. (D) RT-qPCR was conducted to analyze circ_0008450 expression levels in THLE-2, SNU-387 and Huh7 cells ("P<0.05 vs. THLE-2 cells) and to assess the relative expression of circ_0008450 and CMTM3 in Huh7 cells with (E) actinomycin D or (F) RNase R treatment. (G) The expression of circ_0008450 and CMTM3 were measured by RT-qPCR using oligo(dt) $)_{18}$ primers or random primers. ${ }^{*} \mathrm{P}<0.05$. circ, circular; HCC, hepatocellular cancer; CMTM3, CKLF-like MARVEL transmembrane domain containing 3; RT-qPCR, reverse transcription-quantitative PCR; ROC, receiver operating characteristic curve; AUC, area under the curve.
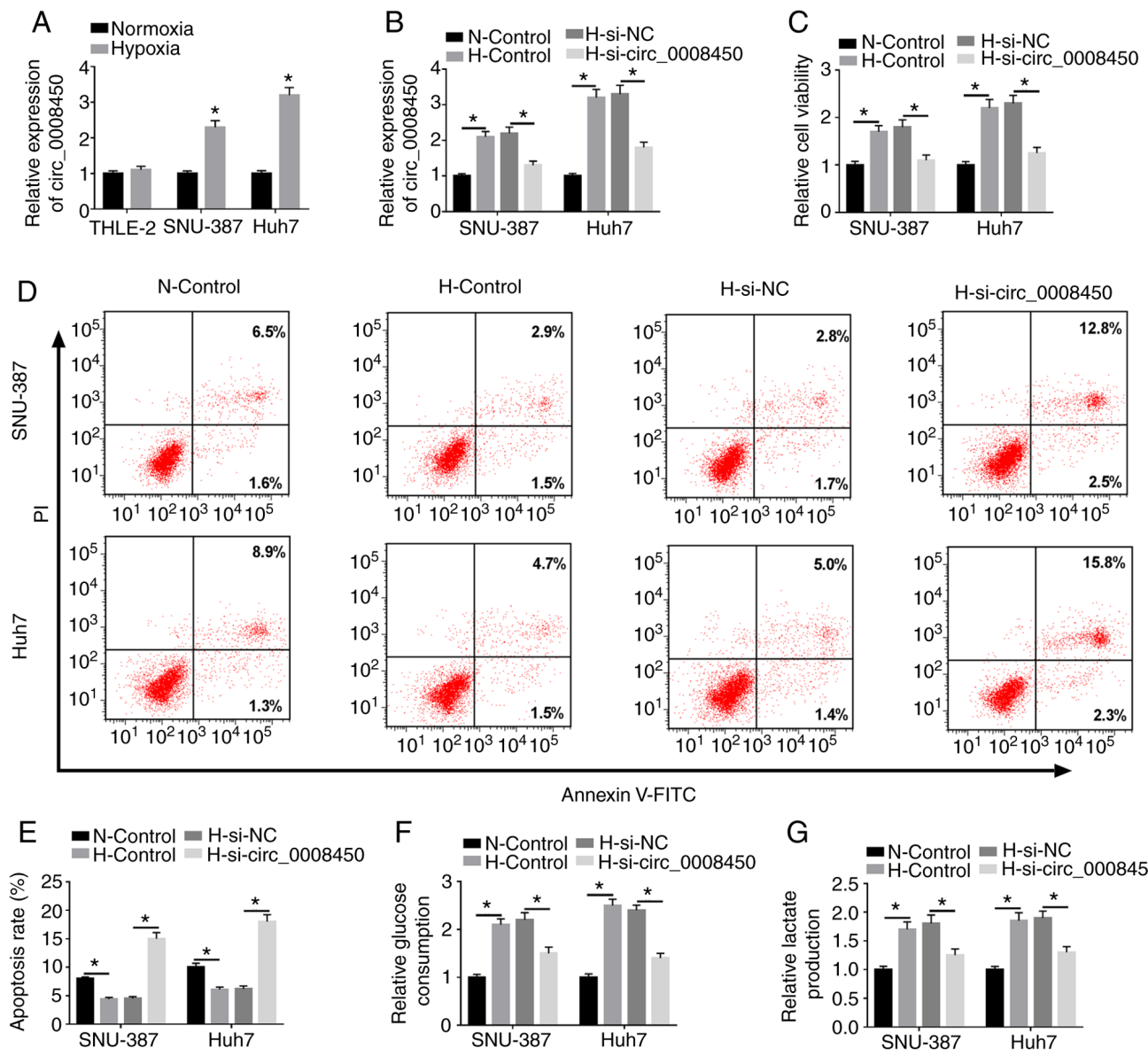

$$
\text { Annexin V-FITC }
$$

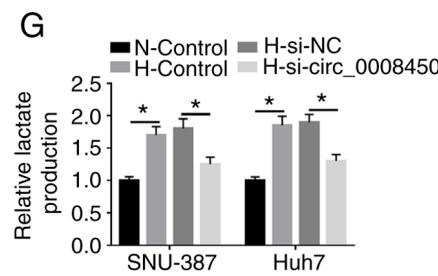

Figure 2. Effects of circ_0008450 inhibition on hypoxia-mediated viability, apoptosis and glycolysis of hepatocellular cancer cells. (A) Effect of hypoxia on the expression of circ_0008450 in THLE-2, SNU-387 and Huh7 cells was assessed via RT-qPCR. "P<0.05 compared with THLE-2 cells. Following transfection with si-NC or si-circ_0008450, SNU-387 and Huh7 cells were treated for $48 \mathrm{~h}$ under hypoxia conditions. (B) Expression of circ_0008450 in SNU-387 and Huh7 cells was detected using RT-qPCR. (C) Viability of SNU-387 and Huh7 cells was determined using Cell Counting kit-8 assays. (D and E) Flow cytometry was performed to analyze the apoptosis rate of SNU-387 and Huh7 cells. Glycolysis assays were executed to measure (F) glucose consumption and (G) lactate production in SNU-387 and Huh7 cells. " $\mathrm{P}<0.05$. circ, circular; RT-qPCR, reverse transcription-quantitative PCR; si-, small interfering; NC, negative control; H, hypoxia; N, normoxia. 
A WT-circ_0008450: 5' UGAGACCACAGCCCACAAGACAG 3' miR-431: 3' ACGUACUGCCGGACGUUCUGU 5'

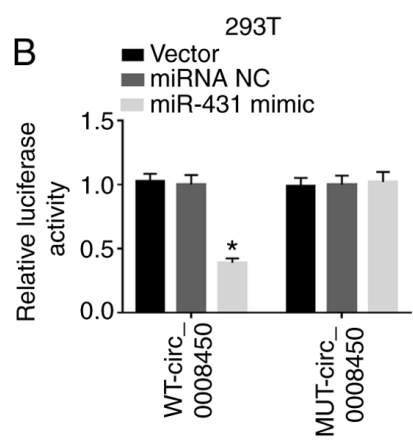
MUT-circ_0008450: 5' UGAGACCACAGCCCAGUUCUGAG 3'
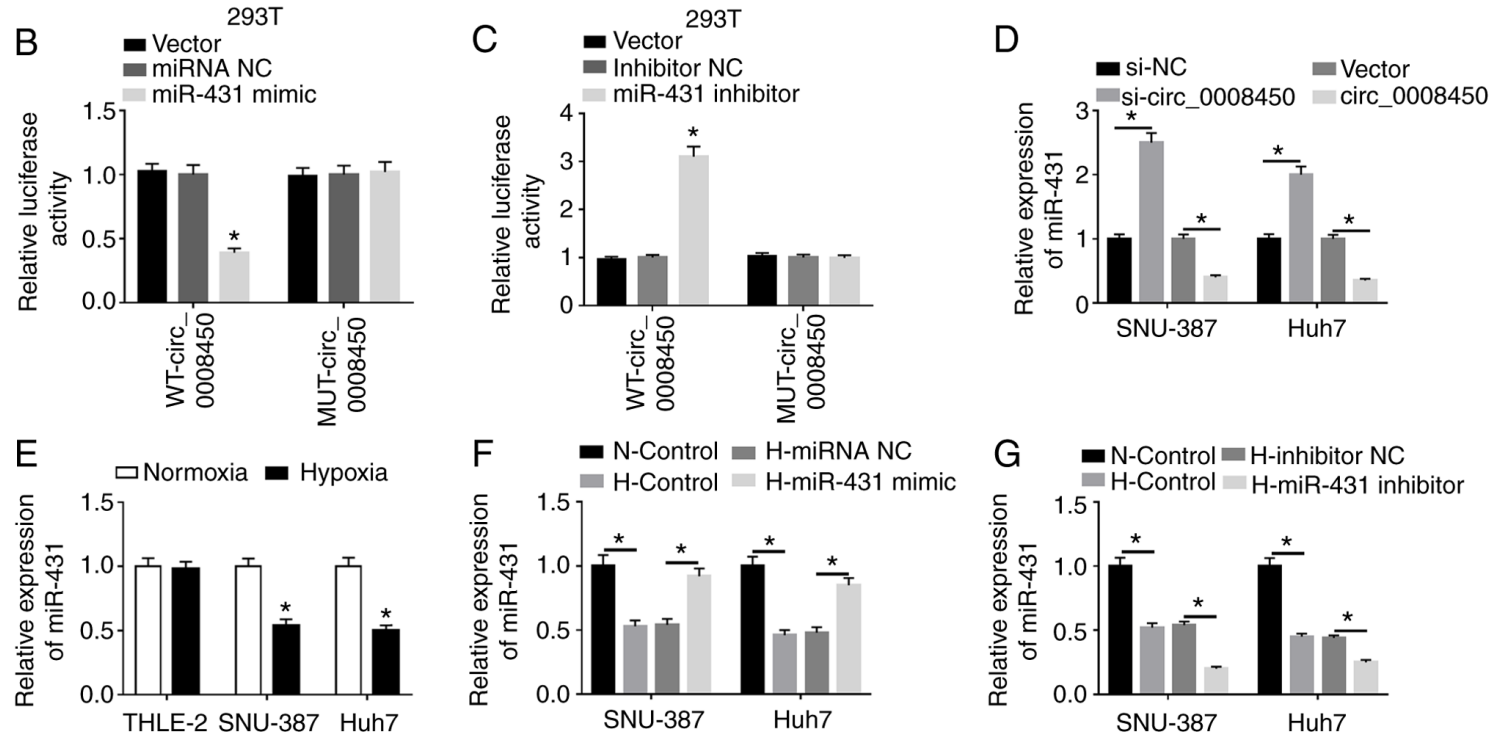

Figure 3. miR-431 acted as a target for circ_0008450 in hepatocellular cancer cells. (A) The binding sites of circ_0008450 in miR-431 were predicted using the CircRNA interactome database. Luciferase intensities of the WT and MUT luciferase reporter vectors of circ_0008450 in $293 \mathrm{~T}$ cells transfected with (B) miRNA NC, miR-431 mimic, (C) inhibitor NC or miR-431 inhibitor were determined by dual-luciferase reporter assays. (D) Effect of circ_0008450 on the expression of miR-431 was assessed using RT-qPCR. (E) The expression of miR-431 in THLE-2, SNU-387 and Huh7 cells treated with hypoxia was detected by RT-qPCR. The transfection efficiency of miR-431 (F) overexpression and (G) silencing on SNU-387 and Huh7 cells treated with hypoxia was evaluated by RT-qPCR. "P<0.05. miR, microRNA; circ, circular; WT, wild-type; MUT, mutant; NC, negative control; RT-qPCR, reverse transcription-quantitative PCR; $\mathrm{N}$, normoxic; $\mathrm{H}$, hypoxic.

vectors (Fig. S5). Furthermore, miR-431 expression was significantly increased by circ_0008450 downregulation and was significantly decreased by circ_0008450 enhancement in SNU-387 and Huh7 cells (Fig. 3D). miR-431 expression was reduced in SNU-387 and Huh7 cells under hypoxia treatment (Fig. 3E). Additionally, miR-431 was significantly upregulated in SNU-387 and Huh7 cells transfected with miR-431 mimic and was significantly downregulated in cells transfected with miR-431 inhibitor under hypoxic conditions (Fig. 3F and G). Overall, these data indicated that circ_0008450 acted as a sponge for miR-431 in HCC.

miR-431 inhibitor reverses circ_0008450 knockdown-mediated effects on viability, apoptosis and glycolysis of HCC cells following hypoxiatreatment. Basedon theabove findings, whether miR-431 was associated with the viability, apoptosis and glycolysis of HCC cells mediated by circ_0008450 following hypoxia was explored. The upregulation of miR-431 in hypoxia-treated SNU-387 and Huh7 cells caused by circ_0008450 silencing was partly recovered by miR-431 inhibition (Fig. 4A). CCK-8 assays demonstrated that the repressive effect of circ_0008450 knockdown on viability of SNU-387 and Huh7 cells under hypoxia treatment was restored by miR-431 suppression (Fig. 4B). Flow cytometry assays revealed that decreased miR-431 expression reversed the increased apoptosis of hypoxia-disposed SNU-387 and Huh7 cells induced by circ_0008450 silencing (Fig. 4C). Additionally, miR-431 knockdown overturned the decrease of glucose consumption and lactate production in SNU-387 and Huh7 cells caused by circ_0008450 depletion under hypoxia treatment (Fig. 4D and E). These results indicated that circ_0008450 mediated the viability, apoptosis and glycolysis of hypoxia-exposed SNU-387 and Huh7 cells by sponging miR-431.

$A K A P 1$ is a target for miR-431. Subsequently, the target for miR-431 was investigated using the Starbase database. The results demonstrated that the 3'UTR of AKAP1 mRNA possessedpossiblebinding sites formiR-431(Fig.5A).Luciferase intensity in $293 \mathrm{~T}$ cells was significantly reduced following cotransfection luciferase reporters containing WT-AKAP1 3'UTR and miR-431 mimic, while the luciferase intensity of luciferase reporters containing MUT-AKAP13'UTR were not significantly different (Fig. 5B). Luciferase intensity of luciferase reporters containing WT-AKAP1 3'UTR was enhanced in miR-431-inhibited 293T cells (Fig. 5C). Moreover, miR-431 mimics significantly decreased the levels of AKAP1 mRNA and protein in SNU-387 and Huh7 cells, while the levels of AKAP1 mRNA and protein were significantly elevated by miR-431 repression (Fig. 5D and E). Additionally, the levels of AKAP1 mRNA were upregulated in SNU-387 and Huh7 cells transfected with pc-AKAP1 compared with control pc-NC (Fig. S5). Furthermore, the mRNA and protein levels of AKAP1 were signally increased in SNU-387 and Huh7 cells following hypoxia (Fig. $5 \mathrm{~F}$ and $\mathrm{G}$ ). In summary, AKAP1 served as a target for miR-431 in HCC cells.

AKAPI overexpression abolishes miR-431 mimic-mediated influence on viability, apoptosis and glycolysis of HCC following hypoxia treatment. Considering miR-431 targeted AKAP1 in HCC cells, whether miR-431 regulated the viability, apoptosis and glycolysis of HCC via AKAP1 following hypoxia treatment was investigated. The results presented that 

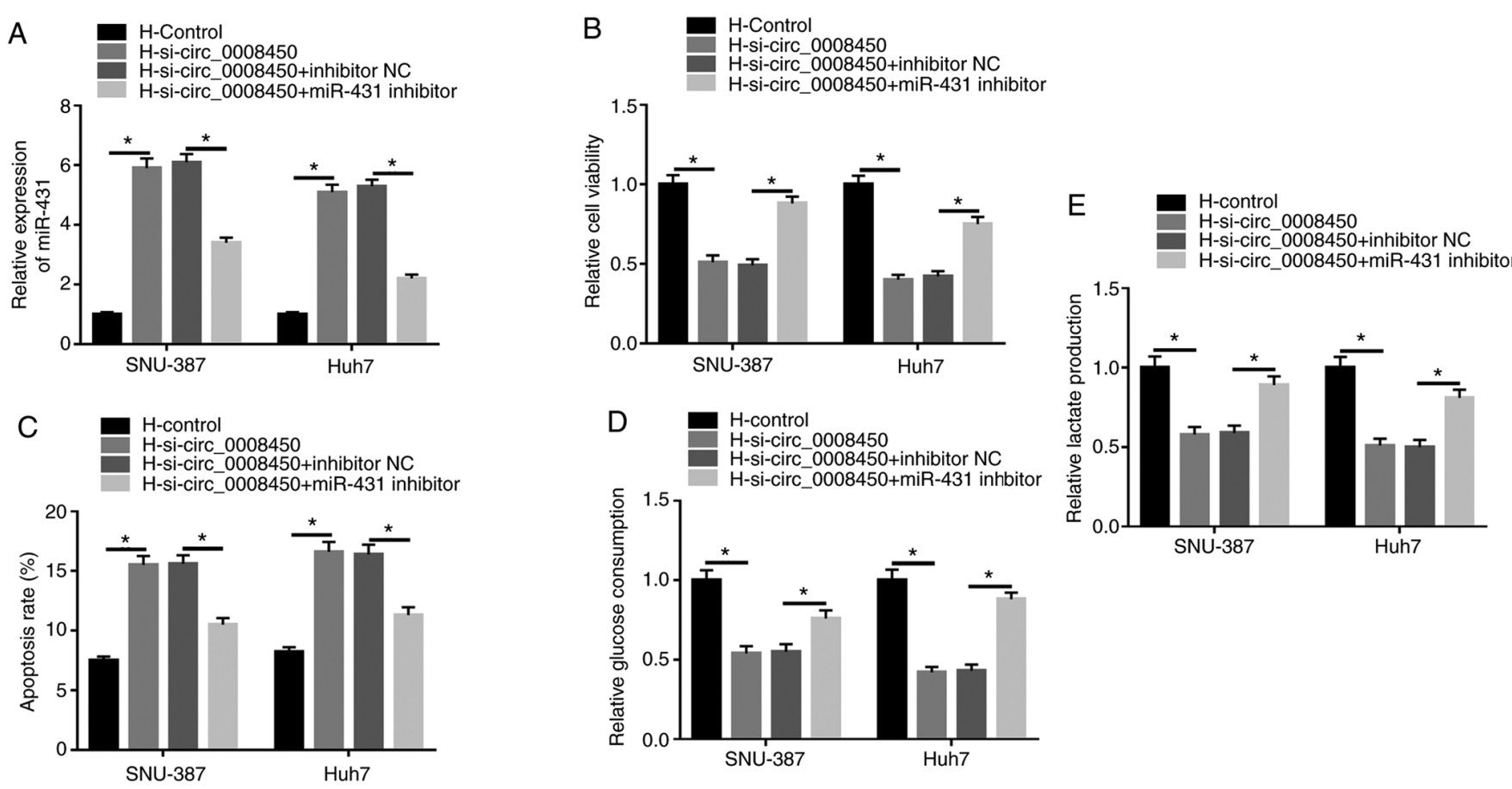

Figure 4. Circ_0008450 regulated the viability, apoptosis and glycolysis of hepatocellular cancer cells via miR-431 following hypoxia treatment. (A) Influence of miR-431 repression on circ_0008450 downregulation-mediated miR-431 expression was analyzed by reverse transcription-quantitative PCR. Effect of miR-431 inhibition on circ_0008450 reduction-mediated (B) viability, (C) apoptosis, (D and E) glycolysis in hypoxia-treated SNU-387 and Huh7 cells were determined using Cell Counting kit-8, flow cytometry and glycolysis assays. " $\mathrm{P}<0.05$. circ, circular; miR, microRNA; si-, small interfering; H, hypoxic; $\mathrm{N}$, normoxic; $\mathrm{NC}$, negative control.

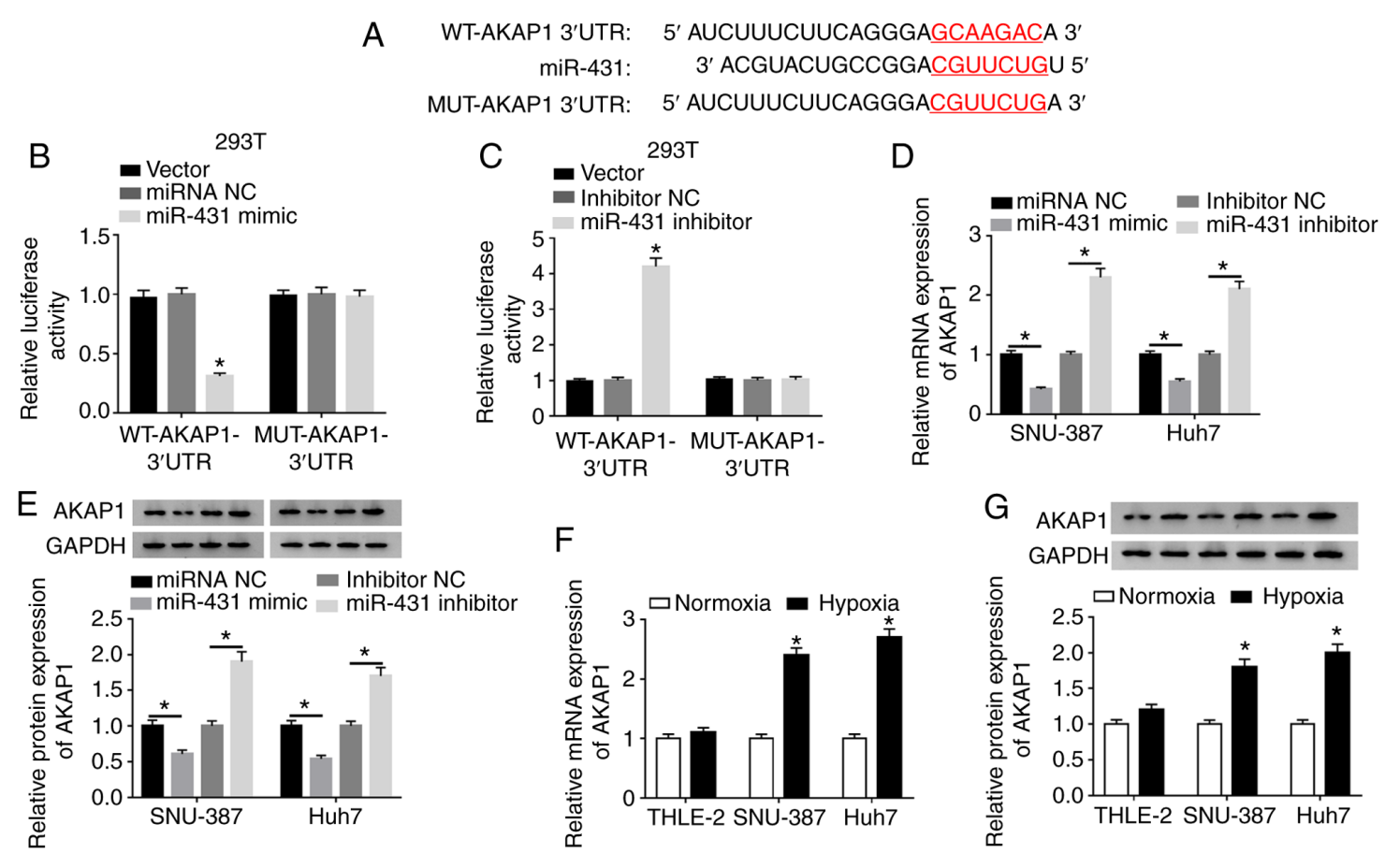

Figure 5. AKAP1 was a target of miR-431 in hepatocellular cancer cells. (A) The binding sites between miR-431 and AKAP1 were predicted using the Starbase database. Dual-luciferase reporter assays were conducted for the evaluation of the luciferase activity in 293T cells contransfected with WT or MUT reporter vectors of AKAP1 and (B) miRNA NC, miR-431 mimic, (C) inhibitor NC or miR-431 inhibitor. Effect of miR-431 on expression of AKAP1 (D) mRNA and (E) protein of SNU-387 and Huh7 cells was analyzed by RT-qPCR and western blot analysis, respectively. Under hypoxic conditions, the (F) mRNA and (G) protein levels of AKAP1 in SNU-387 and Huh7 cells were detected by RT-qPCR and western blot analysis, respectively. "P<0.05. AKAP1, A-kinase anchor protein 1; miR, microRNA; WT, wild-type; MUT, mutant; NC, negative control; RT-qPCR, reverse transcription-quantitative PCR; UTR, untranslated region.

miR-431 overexpression reversed the upregulation of AKAP1 mRNA (Fig. 6A) and protein (Fig. 6B) in hypoxia-treated
SNU-387 and Huh7 cells. Furthermore, AKAP1 overexpression significantly abolished this effect. Increased miR-431 expression 

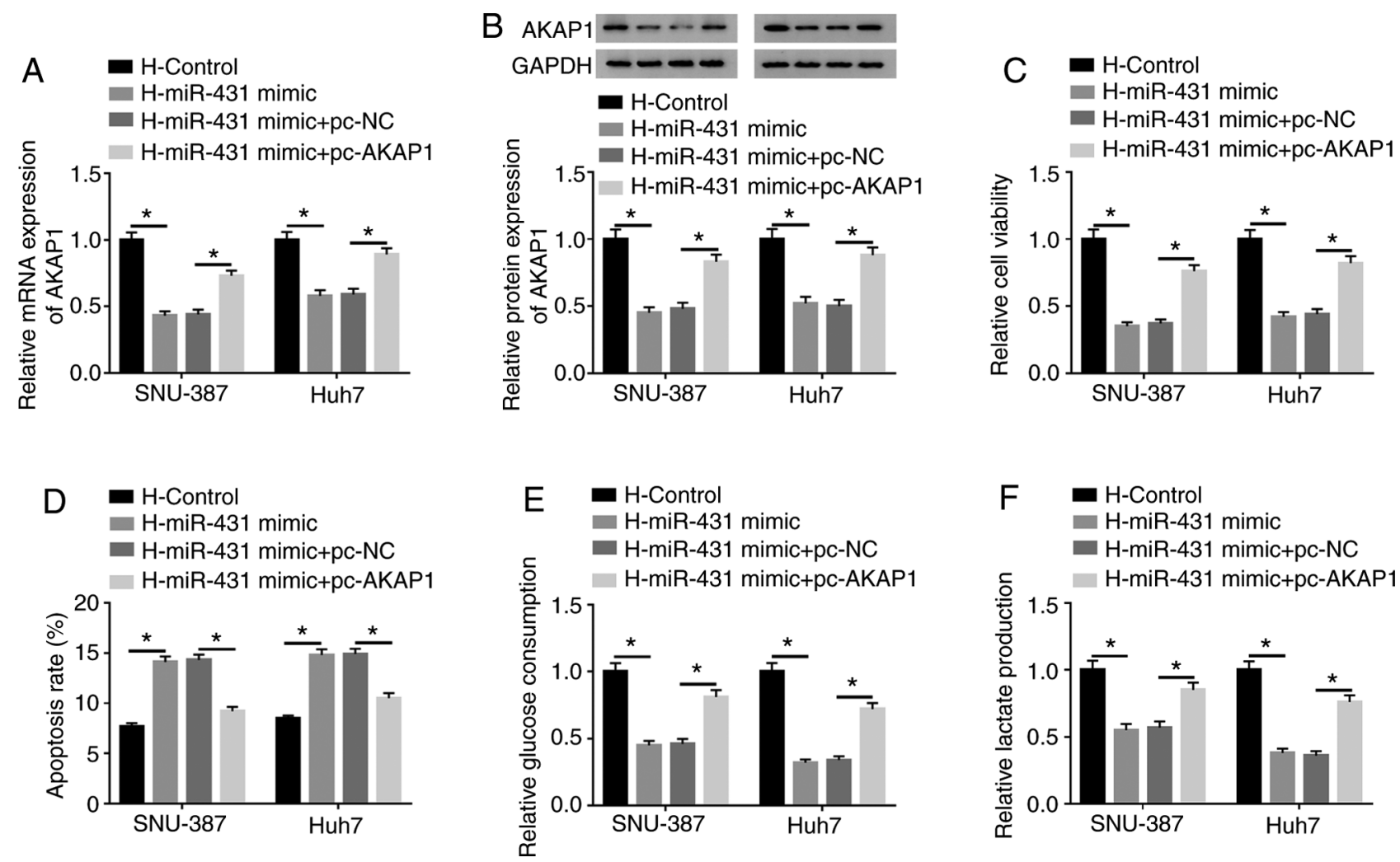

Figure 6. miR-431 modulated viability, apoptosis and glycolysis of hepatocellular cancer cells via AKAP1 under hypoxia treatment. The introduction of pc-AKAP1 on the influence of miR-431 mimic-mediated expression of AKAP1 (A) mRNA and (B) protein of hypoxia-exposed SNU-387 and Huh7 cells was determined with reverse transcription-quantitative PCR and western blotting, respectively. The enhancement of AKAP1 on the impacts of miR-431 mimic-mediated (C) viability, (D) apoptosis and (E and F) glycolysis of hypoxia-disposed SNU-387 and Huh7 cells were evaluated using Cell Counting kit-8, flow cytometry and glycolysis assays. ${ }^{*} \mathrm{P}<0.05$. miR, microRNA; AKAP1, A-kinase anchor protein 1; H, hypoxic; NC, negative control.

A

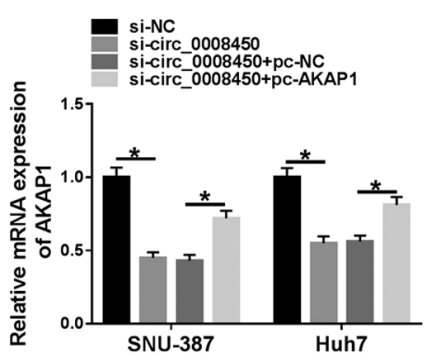

B

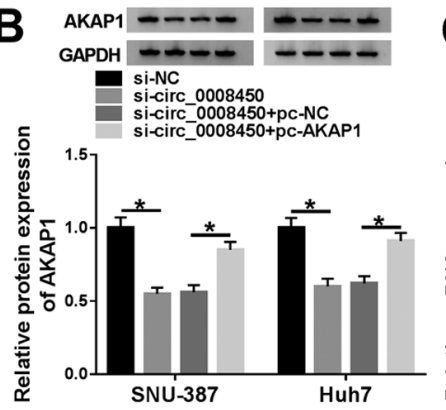

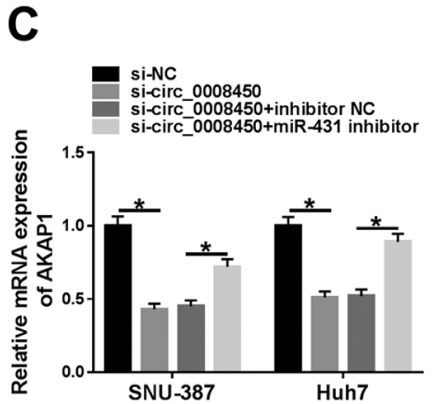

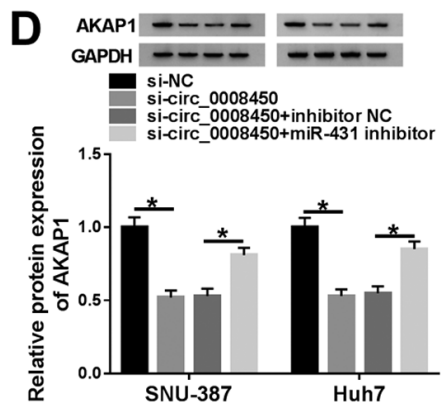

Figure 7. Circ_0008450 regulated AKAP1 expression by miR-431 in hypoxia-disposed SNU-387 and Huh7 cells. (A and B) Effect of AKAP1 enhancement or (C and D) miR-431 inhibitor on circ_0008450 depletion-mediated expression of AKAP1 mRNA and protein of hypoxia-disposed SNU-387 and Huh7 cells was examined using reverse transcription-quantitative PCR and western blotting. "P $<0.05$. Circ, circular; AKAP1, A-kinase anchor protein 1; miR, microRNA; si-, small interfering; NC, negative control.

inhibited cell viability in hypoxia-disposed SNU-387 and Huh7 cells and this repression was overturned by AKAP1 overexpression (Fig. 6C). The results of the flow cytometry assays revealed an elevated apoptosis rate in hypoxia-treated SNU-387 and Huh7 cells caused by increased miR-431 expression was reversed by the introduction of AKAP1 (Fig. 6D). Additionally, the inhibitory impacts of miR-431 upregulation on glucose consumption and lactate production in hypoxia-disposed SNU-387 and Huh7 cells were restored by AKAP1 elevation (Fig. 6E and F). In summary, these results indicated that miR-431 mediated cell viability, apoptosis and glycolysis through AKAP1 in hypoxia-disposed HCC cells.
AKAP1 is modulated by circ_0008450 by sponging miR-431. Considering miR-431 was a target of circ_0008450 and miR-431 targeted AKAP1 in SNU-387 and Huh7 cells, whether circ_0008450 regulated the expression of AKAP1 via miR-431 in SNU-387 and Huh7 cells was explored. The results demonstrated that the mRNA (Fig. 7A) and protein (Fig. 7B) levels of AKAP1 were decreased following the downregulation of circ_0008450 in SNU-387 and Huh7 cells, and that this effect was reversed by AKAP1 overexpression. Moreover, miR-431 inhibitor abolished the inhibitory influence of circ_0008450 silencing on the levels of AKAP1 mRNA (Fig. 7C) and protein (Fig. 7D) of SNU-387 and Huh7 cells. These data 

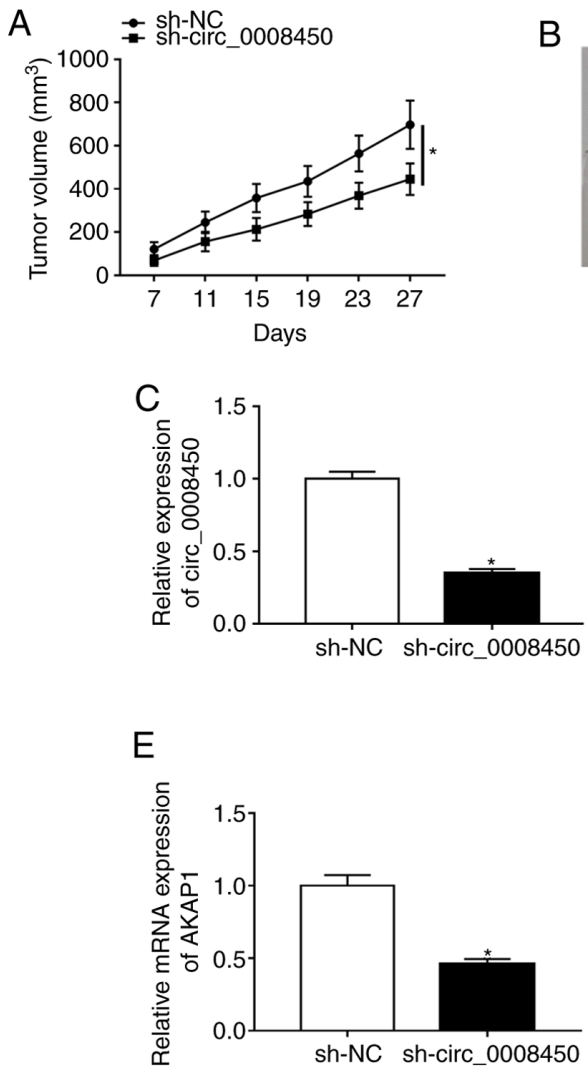
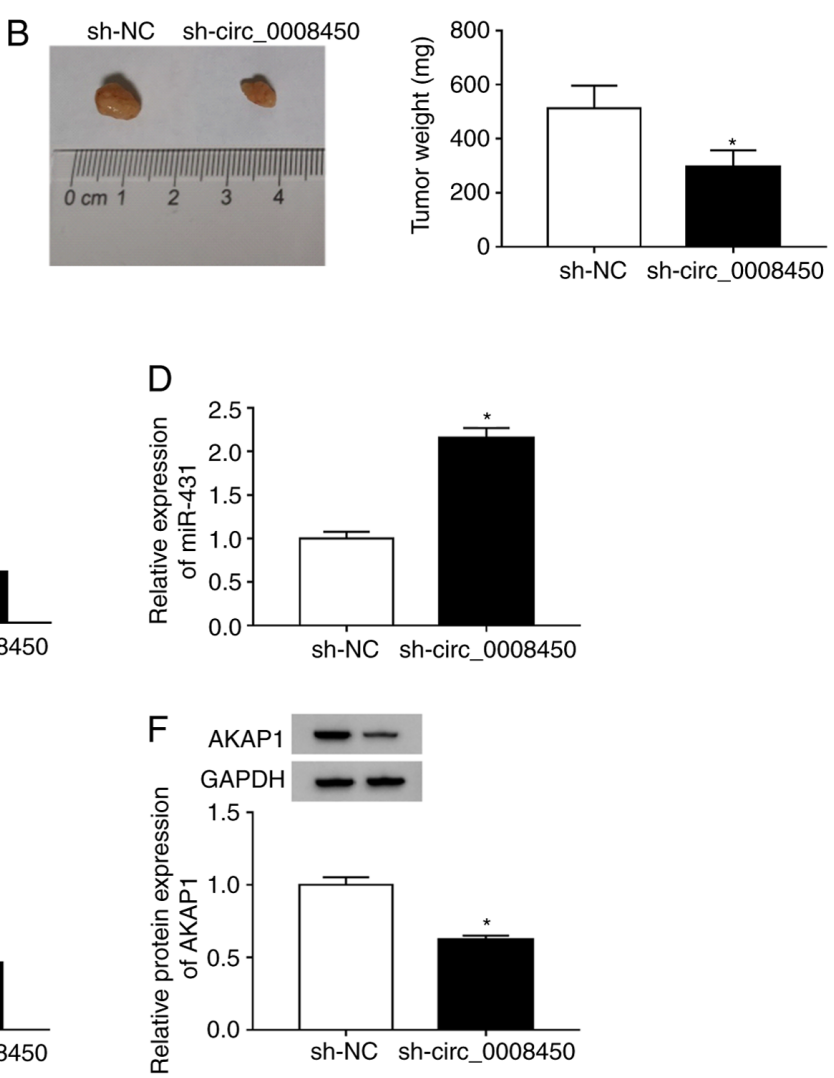

Figure 8. Effect of circ_0008450 on tumor growth in vivo. (A) On day 7, the tumor volumes of the mice in the sh-circ_0008450 and sh-NC groups were measured every 4 days. (B) Tumor weights were assessed on day 27. Expression of (C) circ_0008450 and (D) miR-431 in tumors were detected via RT-qPCR. Expression of AKAP1 (E) mRNA and (F) protein in tumors were examined by RT-qPCR and western blotting, respectively. ${ }^{*} \mathrm{P}<0.05$. Circ, circular; sh, short hairpin; NC, negative control; miR, microRNA; RT-qPCR, reverse transcription-quantitative PCR; AKAP1, A-kinase anchor protein 1.

indicated that circ_0008450 regulated the expression of AKAP1 via miR-431 in SNU-387 and Huh7 cells.

Circ_0008450 depletion reduced HCC growth. To explore the effect of circ_0008450 on HCC tumor growth in vivo, xenograft assay in nude mice was conducted. The results presented that circ_0008450 expression was reduced in Huh7 cells after transfected with sh-circ_0008450 (Fig. S6). Moreover, tumor volumes (Fig. 8A) and weights (Fig. 8B) were reduced in the sh-circ_0008450 group compared to the sh-NC group. Furthermore, circ_0008450 expression was significantly decreased (Fig. 8C) and miR-431expression significantly increased (Fig. 8D) in the sh-circ_0008450 group compared with the sh-NC group. Additionally, AKAP1 mRNA (Fig. 8E) and protein (Fig. 8F) levels were significantly decreased in the sh-circ_0008450 group compared with the control group. Additionally, the levels of caspase-3 were elevated in mice tumor tissues of the sh-circ_0008450 group compared with the sh-NC group (Fig. S7). Collectively, these data demonstrated that circ_0008450 downregulation suppressed tumor growth in vivo.

Hypoxia-induced circ_000845 regulates HCC growth by the miR-431/AKAP1 pathway. Based on the above findings, we concluded that hypoxia-induced circ_000845 elevated AKAP1 expression through competitively binding to miR-431, thereby accelerating proliferation and constraining apoptosis of HCC cells, leading to the growth of HCC (Fig. 9).

\section{Discussion}

The rapid growth of HCC cells usually exceeds the growth of functional blood vessels, which often causes insufficient oxygen delivery in HCC tumors (30). However, in order to provide sufficient energy and substances for the anabolic metabolism of cancer cells, these cells alter their glucose metabolism from oxidative to glycolysis to adapt to hypoxic stress (31). In recent years, circRNAs have been reported to be associated with the progression of hypoxia-mediated tumors. For instance, hypoxia-induced circDENND2A accelerated cell malignancy in glioma cells by sponging miR-625-5p (15). Additionally, circ_0000977 repression elevated the killing effect of natural killer cells in pancreatic cancer cells under hypoxic conditions (32). In the current study, circ_0008450 was upregulated in HCC tissues and hypoxia-treated HCC cells. High circ_0008450 expression was associated with tumor size, TNM stage, lymphatic metastasis and distant metastasis in patients with HCC. Moreover, circulating circ_0008450 had a high diagnostic value. These results indicated that hsa_circ_0084927 may be a novel biomarker for the diagnosis of HCC. Furthermore, circ_0008450 inhibition reduced tumor growth in vivo and reversed its effects on viability and glycolysis, and promoted the apoptosis of hypoxia-disposed HCC cells in vitro. Zhang et al (17) revealed that circ_0008450 downregulation induced cell apoptosis and repressed cell invasion, proliferation and migration in HCC cells. Moreover, Lin et al (33) indicated that circ_0008450 


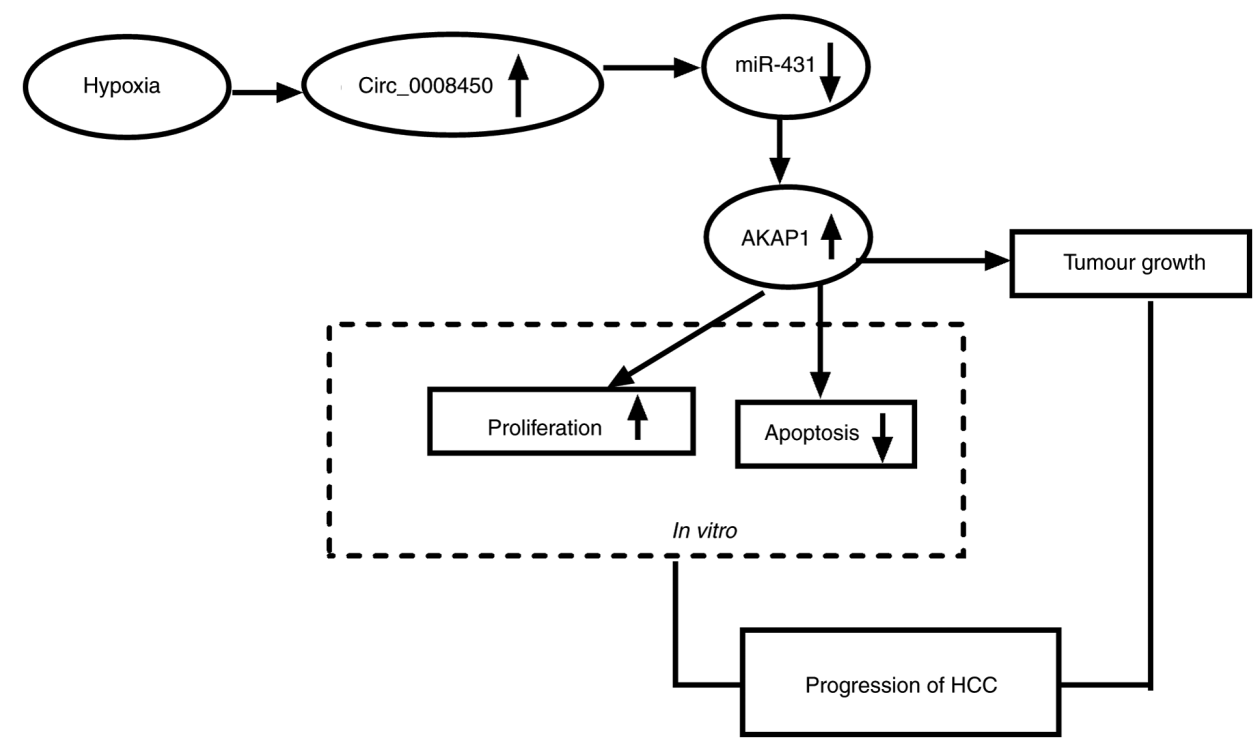

Figure 9. Schematic diagram of the mechanism of circ_0008450 involved in HCC progression. Hypoxia-induced circ_0008450 promoted HCC progression by upregulating AKAP1 via miR-431. circ, circular; HCC, hepatocellular cancer; AKAP1, A-kinase anchor protein 1; miR, microRNA.

knockdown reversed invasion, proliferation and migration of HCC cells. Furthermore, circ_0008450 was revealed to be an oncogene in nasopharyngeal cancer (16). These data indicated that circ_0008450 acted as a potential diagnostic biomarker for HCC and hypoxia-induced circ_0008450 served a cancerogenic role in HCC.

It has been reported that circ_0008450 regulated tumor progression by acting as a sponge for miRNAs $(16,33)$. For instance,hsa_circ_0008450 knockdown impeded the advancement of hepatocellular carcinoma by downregulating enhancer of zeste homolog 2 by sponging miR-214-3p (33). Furthermore, miR-431 was revealed to act as an anti-tumor gene in a variety of cancers. For example, a previous report demonstrated that miR-431 inhibited metastasis and proliferation of lung cancer cells by repressing DEAD box protein expression 5 (22). Another study revealed that silenced miR-431 expression accelerated the invasion of papillary thyroid cancer and was associated with lymph node metastasis (34). Previous studies have demonstrated that miR-431 reversed cell invasion and invasion in HCC cells $(23,35)$. In the current study, miR-431 expression was reduced in HCC cells treated with hypoxia and acted as a target for circ_0008450. Additionally, miR-431 suppression reversed circ_0008450 silencing-mediated effects on the viability, apoptosis and glycolysis of hypoxia-treated HCC cells. These results indicated that hypoxia-induced circ_0008450 modulated the malignant behavior of HCC cells through miR-431 under hypoxic conditions.

As a scaffold protein, AKAP1 has been reported to serve a vital role in controlling mitochondrial function (36). A previous report reported that AKAP1 supported the mTOR pathway and increased the growth of tumor cells (25). Increased AKAP1 expression was associated portal vein thrombosis, tumor size and tumor-mode-metastasis stage of patients with $\mathrm{HCC}$ and AKAP1 may act as a potential prognostic biomarker for the prediction of survival in patients with $\mathrm{HCC}$ followed by radical resection (27). In the current study, AKAP1 was regulated by circ_0008450 via miR-431. AKAP1 elevation reversed the effects of miR-431 on the viability, apoptosis and glycolysis of HCC cells following hypoxia treatment. Therefore, circ_0008450 contributed to HCC progression by upregulating AKAP1 and sponging miR-431 under hypoxia conditions. Unfortunately, only molecular biology research was conducted in the current study and clinical analysis was not performed on the samples. This can be investigated in future studies.

In conclusion, the current study demonstrated that hypoxia-induced circ_0008450 mediated the malignant behavior of HCC cells under hypoxia through the miR-431/AKAP1 axis and circ_0008450 may act as a possible therapeutic target for HCC.

\section{Acknowledgements}

Not applicable.

\section{Funding}

No funding was received.

\section{Availability of data and materials}

The datasets used and/or analyzed during the current study are available from the corresponding author on reasonable request

\section{Authors' contributions}

QD participated in study design, methodology and data interpretation, and drafted the manuscript. JH performed data collection, analysis and statistical analysis. SG participated in the methodology and data interpretation. SZ performed data interpretation and methodology. YP interpreted data. All authors read and approved the final manuscript.

\section{Ethics approval and consent to participate}

The current study was authorized by the Ethics Committee of Lanzhou University Second Hospital, Lanzhou, China. 


\section{Patient consent for publication}

Not applicable.

\section{Competing interests}

The authors declare that they have no competing interests.

\section{References}

1. Bray F, Ferlay J, Soerjomataram I, Siegel RL, Torre LA and Jemal A: Global cancer statistics 2018: GLOBOCAN estimates of incidence and mortality worldwide for 36 cancers in 185 countries. CA Cancer J Clin 68: 394-424, 2018.

2. Forner A, Llovet JM and Bruix J: Hepatocellular carcinoma. Lancet 379: 1245-1255, 2012.

3. El-Serag HB and Rudolph KL: Hepatocellular carcinoma: Epidemiology and molecularcarcinogenesis. Gastroenterology 132 2557-2576, 2007.

4. Zhu P, Ning Y, Yao L, Chen M and Xu C: The proliferation, apoptosis, invasion of endothelial-like epithelial ovarian cancer cells induced by hypoxia. J Exp Clin Cancer Res 29: 124, 2010.

5. Du C, Weng X, Hu W, Lv Z, Xiao H, Ding C, Gyabaah OA, Xie H, Zhou L, Wu J, et al: Hypoxia-inducible MiR-182 promotes angiogenesis by targeting RASA1 in hepatocellular carcinoma. J Exp Clin Cancer Res 34: 67, 2015.

6. Rankin EB and Giaccia AJ: Hypoxic control of metastasis. Science 352: 175-180, 2016

7. Su Y, Yang W, Jiang N, Shi J, Chen L, Zhong G, Bi J, Dong W, Wang Q, Wang C, et al: Hypoxia-elevated circELP3 contributes to bladder cancer progression and cisplatin resistance. Int J Biol Sci 15: 441-452, 2019.

8. Ashwal-Fluss R, Meyer M, Pamudurti NR, Ivanov A, Bartok O, Hanan M, Evantal N, Memczak S, Rajewsky N and Kadener S: circRNA biogenesis competes with pre-mRNA splicing. Mol Cell 56: 55-66, 2014.

9. Ebbesen KK, Hansen TB and Kjems J: Insights into circular RNA biology. RNA Biol 14: 1035-1045, 2017.

10. Zhao ZJ and Shen J: Circular RNA participates in the carcinogenesis and the malignant behavior of cancer. RNA Biol 14: 514-521, 2017.

11. Wang R, Zhang S, Chen X, Li N, Li J, Jia R, Pan Y and Liang H: CircNT5E acts as a sponge of miR-422a to promote glioblastoma tumorigenesis. Cancer Res 78: 4812-4825, 2018.

12. Qiu M, Xia W, Chen R, Wang S, Xu Y, Ma Z, Xu W, Zhang E, Wang J, Fang T, et al: The circular RNA circPRKCI promotes tumor growth in lung adenocarcinoma. Cancer Res 78 2839-2851, 2018.

13. Ren S, Liu J, Feng Y, Li Z, He L, Li L, Cao X, Wang Z and Zhang Y: Knockdown of circDENND4C inhibits glycolysis, migration and invasion by up-regulating $\mathrm{miR}-200 \mathrm{~b} / \mathrm{c}$ in breast cancer under hypoxia. J Exp Clin Cancer Res 38: 388, 2019.

14. Wei Y, Zhang Y, Meng Q, Cui L and Xu C: Hypoxia-induced circular RNA has_circRNA_403658 promotes bladder cancer cell growth through activation of LDHA. Am J Transl Res 11: $6838-6849,2019$

15. Su H, Zou D, Sun Y and Dai Y: Hypoxia-associated circDENND2A promotes glioma aggressiveness by sponging miR-625-5p. Cell Mol Biol Lett 24: 24, 2019.

16. Wei H, Liu D, Sun J, Mao Y, Zhao L, Zhu W, Xu G and Gao Z: Circular RNA circ_0008450 upregulates CXCL9 expression by targeting miR-577 to regulate cell proliferation and invasion in nasopharyngeal carcinoma. Exp Mol Pathol 110: 104288, 2019.

17. Zhang J, Chang Y, Xu L and Qin L: Elevated expression of circular RNA circ_0008450 predicts dismal prognosis in hepatocellular carcinoma and regulates cell proliferation, apoptosis, and invasion via sponging miR-548p. J Cell Biochem 120: 9487-9494, 2019

18. Lu TX and Rothenberg ME: MicroRNA. J Allergy Clin Immunol 141: 1202-1207, 2018.
19. Krol J, Loedige I and Filipowicz W: The widespread regulation of microRNA biogenesis, function and decay. Nat Rev Genet 11: 597-610, 2010.

20. Yang J, Zhu H, Jin Y and Song Y: MiR-431 inhibits cell proliferation and induces cell apoptosis by targeting CDK14 in pancreatic cancer. Eur Rev Med Pharmacol Sci 22: 4493-4499, 2018.

21. Su WB and Liu ZY: MiR-431 inhibits colorectal cancer cell invasion via repressing CUL4B. Eur Rev Med Pharmacol Sci 22: 3047-3052, 2018

22. Xu CM, Chen LX, Gao F, Zhu MF, Dai Y, Xu Y and Qian WX: MiR-431 suppresses proliferation and metastasis of lung cancer via down-regulating DDX5. Eur Rev Med Pharmacol Sci 23: 699-707, 2019.

23. Sun K, Zeng T, Huang D, Liu Z, Huang S, Liu J and Qu Z: MicroRNA-431 inhibits migration and invasion of hepatocellular carcinoma cells by targeting the ZEB1-mediated epithelial-mensenchymal transition. FEBS Open Bio 5: 900-907, 2015.

24. Feliciello A, Gottesman ME and Avvedimento EV: The biological functions of A-kinase anchor proteins. J Mol Biol 308: 99-114, 2001

25. Rinaldi L, Sepe M, Delle Donne R, Conte K, Arcella A, Borzacchiello D, Amente S, De Vita F, Porpora M, Garbi C, et al: Mitochondrial AKAP1 supports mTOR pathway and tumor growth. Cell Death Dis 8: e2842, 2017.

26. Schiattarella GG, Cattaneo F, Carrizzo A, Paolillo R, Boccella N, Ambrosio M, Damato A, Pironti G, Franzone A, Russo G, et al: Akapl regulates vascular function and endothelial cells behavior. Hypertension 71: 507-517, 2018.

27. Yu J, Zhang Y, Zhou D, Wu J and Luo R: Higher expression of A-kinase anchoring-protein 1 predicts poor prognosis in human hepatocellular carcinoma. Oncol Lett 16: 131-136, 2018.

28. Livak KJ and Schmittgen TD: Analysis of relative gene expression data using real-time quantitative PCR and the 2(-Delta Delta C(T)) method. Methods 25: 402-408, 2001.

29. Jiang ZJ, Shen QH, Chen HY, Yang Z, Shuai MQ and Zheng SS: Galectin-1 gene silencing inhibits the activation and proliferation but induces the apoptosis of hepatic stellate cells from mice with liver fibrosis. Int J Mol Med 43: 103-116, 2019.

30. Rankin EB, Nam JM and Giaccia AJ: Hypoxia: Signaling the metastatic cascade. Trends Cancer 2: 295-304, 2016.

31. Zhang X, Li Y, Ma Y, Yang L, Wang T, Meng X, Zong Z, Sun X, Hua X and Li H: Yes-associated protein (YAP) binds to HIF-1a and sustains HIF-1 $\alpha$ protein stability to promote hepatocellular carcinoma cell glycolysis under hypoxic stress. J Exp Clin Cancer Res 37: 216, 2018.

32. Ou ZL and Luo Z: Hypoxia-induced shedding of MICA and HIF1A-mediated immune escape of pancreatic cancer cells from NK cells: Role of circ_0000977/miR-153 axis. RNA Biol 16: 1592-1603, 2019.

33. Lin T, Dai Y, Guo X, Chen W, Zhao J, Cao L and Wu Z: Silencing of hsa_circ 0008450 represses hepatocellular carcinoma progression through regulation of microRNA-214-3p/EZH2 axis. Cancer Manag Res 11: 9133-9143, 2019.

34. Liu Y, Li L, Liu Z, Yuan Q and Lu X: Downregulation of MiR-431 expression associated with lymph node metastasis and promotes cell invasion in papillary thyroid carcinoma. Cancer Biomark 22: 727-732, 2018.

35. Li MF, Li YH, He YH, Wang Q, Zhang Y, Li XF, Meng XM, Huang $\mathrm{C}$ and $\mathrm{Li} \mathrm{J}$ : Emerging roles of hsa circ 0005075 targeting miR-431 in the progress of HCC. Biomed Pharmacother 99: 848-858, 2018.

36. Carlucci A, Adornetto A, Scorziello A, Viggiano D, Foca M, Cuomo O, Annunziato L, Gottesman $\mathrm{M}$ and Feliciello $\mathrm{A}$ Proteolysis of AKAP121 regulates mitochondrial activity during cellular hypoxia and brain ischaemia. EMBO J 27: 1073-1084, 2008 .

This work is licensed under a Creative Commons Attribution-NonCommercial-NoDerivatives 4.0 International (CC BY-NC-ND 4.0) License. 